Mareike Meis

Die Ästhetisierung und Politisierung des Todes

Edition Medienwissenschaft | Band 70 
Mareike Meis, geb. 1982, ist wissenschaftliche Mitarbeiterin am Institut für Friedenssicherungsrecht und Humanitäres Völkerrecht (IFHV) der Ruhr-Universität Bochum und Direktorin des Master-Studienganges Internationale Humanitäre Hilfe im Network on Humanitarian Action (NOHA). Die Forschungsschwerpunkte der kulturwissenschaftlich ausgerichteten Medienwissenschaftlerin sind die Ästhetik neuer Medien, die Rolle der Medien in Krieg, Konflikt und Genozid, die Wahrnehmung des Todes im Kontext neuer Medien, soziale Bewegungen im Mittleren Osten und Nordafrika sowie die Sozialpsychologie der Gewalt. 
Mareike Meis

\section{Die Ästhetisierung und Politisierung des Todes}

Handyvideos von Gewalt und Tod im Syrienkonflikt 
Angenommen als Dissertation zur Erlangung des Grades eines Doktors der Philosophie in der Fakultät für Philologie der Ruhr-Universität Bochum unter Begutachtung von Prof. Dr. Astrid Deuber-Mankowsky und Prof. Dr. Eva Warth.

Datum der Disputation: 05.07.2019

Gedruckt mit freundlicher Unterstützung der Geschwister Boehringer Ingelheim Stiftung für Geisteswissenschaften in Ingelheim am Rhein.

Diese Publikation wurde im Rahmen des Fördervorhabens 16TOA002 mit Mitteln des Bundesministeriums für Bildung und Forschung im Open Access bereitgestellt.

\section{Bibliografische Information der Deutschen Nationalbibliothek}

Die Deutsche Nationalbibliothek verzeichnet diese Publikation in der Deutschen Nationalbibliografie; detaillierte bibliografische Daten sind im Internet über http://dnb.d-nb.de abrufbar.

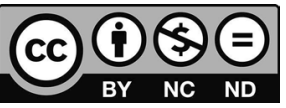

Dieses Werk ist lizenziert unter der Creative Commons Attribution-NonCommercial-NoDerivs 4.0 Lizenz (BY-NC-ND). Diese Lizenz erlaubt die private Nutzung, gestattet aber keine Bearbeitung und keine kommerzielle Nutzung. Weitere Informationen finden Sie unter https://creativecommons.org/licenses/by-nc-nd/4.o/deed.de

Um Genehmigungen für Adaptionen, Übersetzungen, Derivate oder Wiederverwendung zu kommerziellen Zwecken einzuholen, wenden Sie sich bitte an rights@transcript-publishing.com

Die Bedingungen der Creative-Commons-Lizenz gelten nur für Originalmaterial. Die Wiederverwendung von Material aus anderen Quellen (gekennzeichnet mit Quellenangabe) wie z.B. Schaubilder, Abbildungen, Fotos und Textauszüge erfordert ggf. weitere Nutzungsgenehmigungen durch den jeweiligen Rechteinhaber.

\section{(ㄷ) 2021 transcript Verlag, Bielefeld}

Umschlaggestaltung: Maria Arndt, Bielefeld

Druck: Majuskel Medienproduktion GmbH, Wetzlar

Print-ISBN 978-3-8376-5080-8

PDF-ISBN 978-3-8394-5080-2

EPUB-ISBN 978-3-7328-5080-8

https://doi.org/10.14361/9783839450802

Gedruckt auf alterungsbeständigem Papier mit chlorfrei gebleichtem Zellstoff. Besuchen Sie uns im Internet: https://www.transcript-verlag.de Unsere aktuelle Vorschau finden Sie unter www.transcript-verlag.de/vorschau-download 